\title{
Assessment on rheological and texture properties of xylitol-substituted dadih
}

\begin{abstract}
Dadih is a dairy-based dessert made of milk, water, sugar and agar, which is popular especially among South East Asian people. It has sweet taste due to high sugar content in its formulation. An excessive intake of sugar is harmful to human health, and it would be beneficial if the conventional sugar is replaced by a sugar substitute. In order to formulate a healthier dadih, xylitol was used to replace sucrose in three different levels of compositions $(0,50$ and $100 \%)$, which xylitol provides intense sweetness with fewer calories. Therefore, the objective of this study was to evaluate the effect of xylitol substitution on rheological and textural properties of xylitol-substituted dadih. Process parameters were cooking temperatures (85-95C) and cooking times (10-20 min). It was found that cooking temperature had the biggest impact on dadih. All samples showed gel-like behavior where the value of $\mathrm{G}^{\prime}$ (storage modulus) was higher than $\mathrm{G}^{\prime \prime}$ (loss modulus). Samples that were prepared under high temperatures illustrated higher value of $\mathrm{G}^{\prime}$ with elastic gel property regardless of the xylitol composition. Sample prepared at 95C cooking temperature, 20-min cooking time and $0 \%$ xylitol exhibited the highest value of hardness and internal gel strength with $0.892 \mathrm{~N} \pm 0.002$ and $0.248 \mathrm{~N} \pm 0.006$, respectively. In general, the presence of xylitol in dadih contributed to its softer texture compared to a product that is made from sucrose. However, xylitol-substituted dadih prepared at $85 \mathrm{C}$ with longer cooking time (15 to $20 \mathrm{~min}$ ) met the required texture as conventional dadih.
\end{abstract}

Keyword: Dadih; Xylitol; Xylitol-substituted; Rheological; Textural properties 